\title{
Variable Unstressed Volume Keeps Normal Distributions of Canine Left Ventricular Contractility and Total Mechanical Energy under Atrial Fibrillation
}

\author{
Satoshi MOHRI*, Juichiro SHIMIZU*,‡, Haruo ITO*,‡, Hiroki YAMAGUCHI ${ }^{\dagger}$, \\ Shunji SANO ${ }^{\dagger}$, Miyako TAKAKI ${ }^{\ddagger}$, and Hiroyuki SUGA ${ }^{*}, \S$
}

Departments of Cardiovascular *Physiology and +Surgery, Okayama University Graduate School of Medicine, Dentistry and Pharmaceutical Sciences, Shikatacho, Okayama, 700-8558 Japan; :Department of Physiology II, Nara Medical University, Shijocho, Kashihara, Nara, 634-8521; and \$National Cardiovascular Center Research Institute, Fujishirodai, Suita, Osaka, 565-8565 Japan

\begin{abstract}
We have reported that the contractility index $\left(E_{\max }\right)$ and the total mechanical energy (PVA) of arrhythmic beats of the left ventricle (LV) distribute normally in canine hearts under electrically induced atrial fibrillation (AF). Here, $E_{\max }$ is the ventricular elastance as the slope of the end-systolic (ES) pressure-volume $(\mathrm{P}-\mathrm{V})$ relation (ESPVR), and PVA is the systolic P-V area as the sum of the external mechanical work within the P-V loop and the elastic potential energy under the ESPVR. To obtain $E_{\max }$ and PVA, we had to assume the systolic unstressed volume $\left(V_{0}\right)$ as the V-axis intercept of the ESPVR to be constant despite the varying $E_{\max }$, since there was no method to obtain $V_{\text {o }}$ directly in each arrhythmic beat. However, we know that in regular stable beats $V_{\text {o }}$ decreases by $\sim 7 \mathrm{ml} / 100 \mathrm{~g} \mathrm{LV}$ with $\sim 100$
\end{abstract}

times the increases in $E_{\text {max }}$ from $\sim 0.2 \mathrm{mmHg} /$ $(\mathrm{ml} / 100 \mathrm{~g} \mathrm{LV})$ of almost arresting weak beats to $\sim 20 \mathrm{mmHg} /(\mathrm{ml} / 100 \mathrm{~g} \mathrm{LV})$ of strong beats with a highly enhanced contractility. In the present study, we investigated whether $E_{\max }$ and PVA under AF could still distribute normally, despite such $E_{\max }$-dependent $V_{0}$ changes. The present analyses showed that the $E_{\max }$ changes were only $\sim 3$ times at most from the weakest to the strongest arrhythmic beat under AF. These changes were not large enough to affect $V$ enough to distort the frequency distributions of $E_{\max }$ and PVA from normality. We conclude that one could practically ignore the slight $E_{\max }$ and PVA changes with the $E_{\max }$-dependent $V_{0}$ changes under AF. [The Japanese Journal of Physiology 55: 255-264, 2005]

Key words: arrhythmia, cardiac function, $E_{\max }$, PVA, frequency distribution.

Many groups, including ours, have investigated the statistical characteristics of cardiodynamic variables of ventricular arrhythmic beats under atrial fibrillation (AF) in basic and clinical studies [1-17]. We have found that the frequency distributions of the heart rate (HR), beat-to-beat interval (RR), and effective afterload elastance (Ea) (Fig. 1) of the left ventricle (LV) $[18,19]$ are significantly non-normal or nonGaussian in canine hearts under AF [9-11]. However, we have recently found that the frequency distributions of both contractility index $\left(E_{\max }\right)$ and total mechanical energy (PVA) (Fig. 1) are normal or Gaussian in canine LVs under AF $[9-11,14,17]$. Here, $E_{\max }$ is the ventricular elastance as the slope of the end-systolic pressure-volume (P-V) relation (ESPVR) line, and PVA is the systolic P-V area as the sum of the external mechanical work (EW) within the P-V loop and the elastic potential energy (PE) under the ESPVR (Fig. 1). We have attributed this normal distribution of PVA to the central limit theorem by which the complexity of the PVA formula could attenuate the effect of the considerably non-normal distribution of Ea on PVA [10]. 


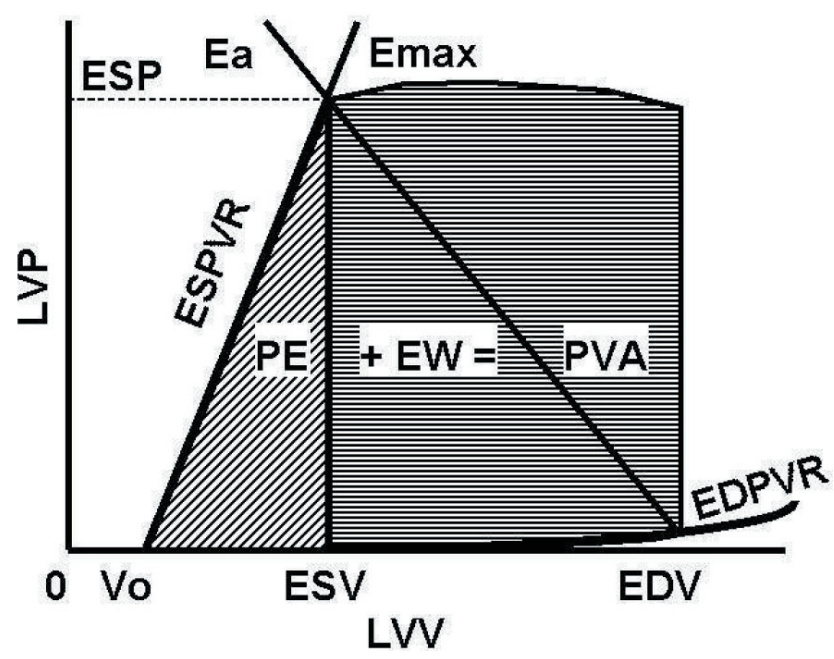

Fig. 1. Schematic illustration of cardiac mechanoenergetic variables in the left ventricular (LV) pressure (P)-volume (V) diagram. ESP $=$ end-systolic pressure. $\mathrm{ESV}=$ end-systolic volume. ESPVR = end-systolic P-V relation. $E_{\max }=$ end-systolic maximum elastance or $\mathrm{P}-\mathrm{V}$ ratio as the slope of ESPVR, which was proposed by Suga and has generally been recognized as a reasonably load-independent index of ventricular contractility. EDV = end-diastolic volume. EDPVR = end-diastolic P-V relation. EW (dotted area) $=$ external mechanical work as the area within the P-V loop. PE (hatched area) = elastic potential energy within the ventricular wall generated by each contraction. PVA = total mechanical energy of each contraction as the sum of EW and PE. $E_{\mathrm{a}}=$ effective arterial elastance. For more details of $E_{\text {max }}, P E$, PVA, and $E_{a}$, refer to Refs. 18,21 , and 23.

The tacit assumption in our previous study [10], however, was that the V-axis intercept $\left(V_{\mathrm{o}}\right)$ of the LV ESPVR was constant despite the arrhythmic beat-bybeat changes in $E_{\max }$ under AF. We had to assume so because $V_{0}$ could not be determined in each arrhythmic beat [10], unlike in the case of regular stable beats at a given $E_{\text {max }}[18,20,21]$. In fact, our earlier study has shown $V_{0}$ to decrease by as much as $\sim 7 \mathrm{ml} / 100$ g LV, but not constantly, with wide increases in $E_{\max }$ [22].

Therefore, in the present study, we investigated quantitatively the magnitude of the influence of the $E_{\max }$-dependent $V_{\text {o }}$ changes on the frequency distributions of both $E_{\max }$ and PVA [18, 21, 23] under AF. The LV P-V data that we used in the present study were the same that we used in the previous study [10]. We newly obtained statistical evidence that the Emax-dependent $V_{\mathrm{o}}$ changes under AF little affected both $E_{\max }$ and PVA and kept their frequency distributions normal or Gaussian. The reason for these results was that the range of arrhythmic changes in $E_{\max }$ under AF was too small to shift $V_{0}$ to the extent that the frequency distributions of both $E_{\text {max }}$ and PVA were deviated from

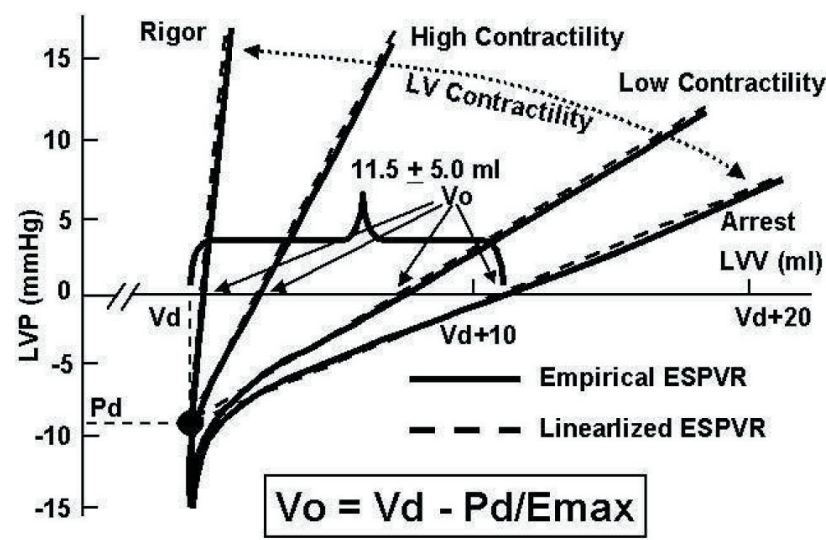

Fig. 2. Experimental evidence for the $E_{\max }$ dependency of the V-axis intercept of the P-V relation (ESPVR), drawn on the basis of our previous study [22]. Thick solid curves show the actual ESPVR curves obtained in rigor (steepest), at a high $E_{\max }$ (second steepest) and a low $E_{\max }$ (second gentle), and under ischemic arrest (most gentle). Thick dashed lines on or near the actual ESPVR curves show their linealized ESPVRs, apparently converging to a common $V_{d}-P_{d}$ point. $V_{d}$ (ventricular dead volume) $=4.1 \pm 1.3(\mathrm{SD})$ $\mathrm{ml} / 100 \mathrm{~g} \mathrm{LV}$ and $P_{\mathrm{d}}=-9.5 \pm 2.0$ (SD) $\mathrm{mmHg}$ [22]. The inset square shows the equation of the $V$-axis intercepts ( $V_{0}$ 's) of these linearized ESPVRs as a function of $E_{\max }$ with $V_{d}$ and $P_{d}$ as parameters as proposed previously [22]. Left ventricu$\operatorname{lar}(\mathrm{LV}) \mathrm{V}$ is labeled in excess of $V_{\mathrm{d}}$. The horizontal brace shows the full working range of $V_{0}$ between the maximum $E_{\max }$ in rigor and minimum $E_{\max }$ under arrest. See Figs. 3-9 of Ref. 22 for more details.

normality. This evidently corroborates our previous conclusion [10].

\section{METHODS}

Theoretical considerations. Figure 2 shows the $E_{\max }$-dependent $V_{\mathrm{o}}$ changes that we had obtained experimentally in canine regularly beating LVs in our earlier study [22]. Here, the four thick solid curves are the actual ESPVR curves for different $E_{\max }$ levels varied from a physiologically high contractile level first down to a low contractile level, further down to the zero contractile level under ischemic arrest, and finally up to the maximum contractile level in rigor. All ESPVR curves were drawn within a low $P(< \pm 15$ $\mathrm{mmHg}$ ) range to see their details around the $\mathrm{V}$ axis. These ESPVR curves merged at a common dead volume $\left(V_{\mathrm{d}}\right)$ below a negative $P$ of $10-15 \mathrm{mmHg}$ [22].

Figure 2 also shows the four ESPVR (thick dashed) lines fitted to the main linear parts of the four ESPVR curves. Although the ESPVR curves at low $E_{\text {max }}$ levels are obviously convex upward in the further negative $P$ range, these curved parts are not observable 

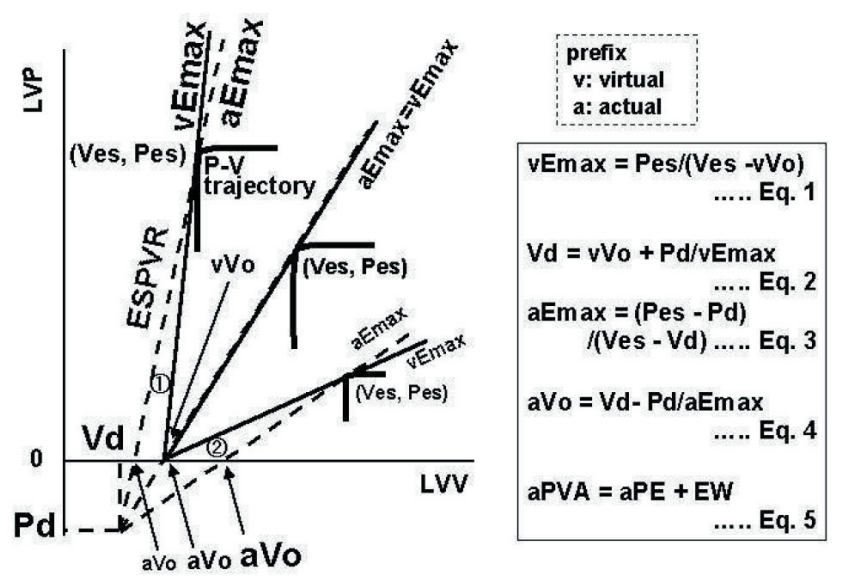

Fig. 3. Schematic illustration of the $E_{\text {max }}$ dependency of $V_{\text {。 }}$ (V-axis intercept of ESPVR line or $E_{\max }$ line) and the resultant deviations of $V_{\mathrm{o}}$ and ${ }_{\mathrm{Emax}}$ from their virtual values ( $v V_{\circ}$ and $\left.v E_{\max }\right)$ to actual values $\left(\mathrm{a} V_{\mathrm{o}}\right.$ and $\left.\mathrm{a} E_{\max }\right)$. The end-systolic (ES, or es) corners of the three P-V loops are shown in the LV P-V diagram. The three solid ESPVR lines passing these ES corners have the same $V$ intercept at $\vee V$ that has been obtained as the LVV-axis intercept by extrapolating the ESPVR of regular stable contractions. However, the actual ESPVR lines at different $\mathrm{aE}_{\max }$ levels rather converge to a single negative $P$ point $\left(V_{d}, P_{d}\right)$. In other words, all the actual ESPVR lines change their slopes or actual $E_{\max }$ $\left(a E_{\text {max }}\right)$ values by rotating around the $V_{d}-P_{d}$ point as a fulcrum. The three dashed ESPVR lines are the actual ESPVR lines with $a E_{\max }$ and $E_{\max }$-dependent actual $V_{0}\left(a V_{0}\right)$. The middle solid and dashed ESPVR lines are drawn to be superimposable on each other, since the extrapolation of the solid ESPVR line passes through the $V_{d}-P_{d}$ point with the same $a E_{\max }$ as the $\vee E_{\max }$ at an intermediate level of $E_{\max }$. The inset square lists 5 equations to describe $\vee E_{\max }$ (Eq. 1), $V_{\mathrm{d}}$ (Eq. 2), a $E_{\max }$ (Eq. 3), a $V_{\circ}$ (Eq. 4), and aPVA (Eq. 5) used in the text. The three resultant a $V$ 's are shown on the LVV axis. As the result, the $\mathrm{a} E_{\max }$ greater than the middle $\mathrm{a} E_{\max }(=\vee E-$ ${ }_{\text {ax }}$ ) is smaller than its corresponding $\vee E_{\text {max }}$, and decreases $\mathrm{PE}$ (elastic potential energy, see Fig. 1) from its vPE to aPE (though not shown) by the triangular area (1). On the contrary, the $\mathrm{a} E_{\max }$ smaller than the middle aEmax $\left(=\mathrm{v} E_{\max }\right)$ is greater than its corresponding $\vee E_{\text {max }}$, and increases $P E$ from its $\mathrm{VPE}$ to aPE (though not shown) by the triangular area (2). Although neither are shown, EW (external mechanical work, see Fig. 2) remains unchanged despite the difference between $\mathrm{a} E_{\max }$ and $\mathrm{v} E_{\max }$.

in normally loaded contractions. These dashed ESPVR lines virtually converged to a common point, the $V$ of which is an LV dead volume $\left(V_{\mathrm{d}}\right)$ and the $P$ of which is an LV convergent pressure $\left(P_{\mathrm{d}}\right)$. Our earlier study has shown $V_{\mathrm{d}}=4.1 \pm 1.3(\mathrm{SD}) \mathrm{ml} / 100 \mathrm{~g} \mathrm{LV}$ and $P_{\mathrm{d}}=-9.5$ $\pm 2.0 \mathrm{mmHg}$ [22].

Our earlier study [22] has also shown that although $E_{\max }$ decreased from a physiologically high $\sim 20$ $\mathrm{mmHg} /(\mathrm{ml} / 100 \mathrm{~g} \mathrm{LV})$ down to a physiologically low $\sim 5 \mathrm{mmHg} /(\mathrm{ml} / 100 \mathrm{~g} \mathrm{LV}), V_{\mathrm{o}}$ increased only by $\sim 1-2$ $\mathrm{ml} / 100 \mathrm{~g} \mathrm{LV}$. However, when $E_{\max }$ further decreased to $\sim 1-0.5 \mathrm{mmHg} /(\mathrm{ml} / 100 \mathrm{~g} \mathrm{LV}), V_{\mathrm{o}}$ sensitively increased to as much as $\sim 3-10 \mathrm{ml} / 100 \mathrm{~g} \mathrm{LV}$. In contrast, when $E_{\text {max }}$ was maximally raised to $\sim 50 \mathrm{mmHg} /(\mathrm{ml} / 100 \mathrm{~g}$ $\mathrm{LV}), V_{\text {o }}$ was as small as $\sim 0.5 \mathrm{ml} / 100 \mathrm{~g} \mathrm{LV}$. As a whole,

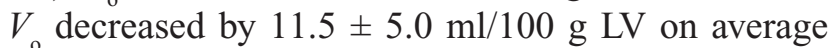
from arrest to rigor in the six LVs, as shown by the horizontal brace (Fig. 2).

These nonlinear relations between $V_{\mathrm{o}}$ and $E_{\max }$ once $V_{\mathrm{d}}$ and $P_{\mathrm{d}}$ are given could theoretically be formulated by the following equation:

$$
V_{\mathrm{o}}=V_{\mathrm{d}}-P_{\mathrm{d}} / E_{\max }
$$

Here, the assumption is that the linearized ESPVR rotates with $E_{\max }$ around the fixed $V_{\mathrm{d}}-P_{\mathrm{d}}$ point as a fulcrum. We assumed these basic concepts of $V_{\mathrm{o}}$ and $V_{\mathrm{d}}$ to hold even in arrhythmic contractions with beat-bybeat variable $E_{\max }$ levels. We then adopted the above equation (see also the inset of Fig. 2) to calculate the varying $V_{\mathrm{o}}$ with $E_{\max }$ of each arrhythmic beat in the present study.

Figure 3 schematically shows three P-V loop segments around the end systole (ES) and two pairs of three ESPVR lines (solid and dashed diagonal). Of these, the solid ESPVR lines have a common fulcrum at the virtual $(\mathrm{v}) V_{\mathrm{o}}\left(\mathrm{v} V_{\mathrm{o}}\right)$ on the $\mathrm{V}$ axis. Here, the $\mathrm{v} V_{\mathrm{o}}$ stands for the conventional, virtually constant or $E_{\max }-$ independent $V_{0}$ obtained in control $E_{\max }$ by clamping the inferior vena cava under regular beats as usual $[10$, $11,18,20,21,23] . E_{\max }$ values obtained using the $\mathrm{v} V$ and virtual ESPVR (vESPVR) as usual are hereafter called virtual $E_{\max }\left(\mathrm{v} E_{\max }\right)$. The $\mathrm{v} V_{\mathrm{o}}$ has usually been used to calculate both $\mathrm{v} E_{\max }$ and virtual PVA (vPVA), even in different $E_{\max }$ levels from that used to obtain $\mathrm{v} V$, as in the case of our previous study [10].

In contrast to these virtual values, the dashed ESPVR lines have a common fulcrum at the $V_{\mathrm{d}}-P_{\mathrm{d}}$ point below the $\mathrm{V}$ axis (Fig. 3). These dashed lines are the actual (a) ESPVR (aESPVR) lines, different from the vESPVR lines, and thus the former lines have different $E_{\text {max }}$ values (actual $E_{\text {max }}, \mathrm{a} E_{\text {max }}$ ) from the corresponding $\mathrm{v} E_{\max }$ 's, though they pass virtually the same end-systolic P-V (Pes-Ves) points. The only exceptional case is the identical $E_{\text {max }}$ of the dashed and solid ESPVR lines, both with the same $\mathrm{v} V_{\mathrm{o}}$ and $\mathrm{a} V_{\mathrm{o}}$, as illustrated by the middle pair of the dashed and solid ESPVR lines. The aESPVR lines intersect the $\mathrm{V}$ axis at different points such as the three $\mathrm{v} V_{0}$ 's in different font sizes (Fig. 3). These intercepts indicate the $\mathrm{a} V$ owich is dependent on aEmax

Figure 3 shows that, as the results, $\mathrm{a} E_{\max }$ is smaller than $\mathrm{v} E_{\text {max }}$ at a greater $E_{\text {max }}$ level than control and 
Table 1. Minor effects of $E_{\max }$ dependency of $V_{\circ}$ on $E_{\max }, \mathrm{PE}$, and PVA.

\begin{tabular}{|c|c|c|c|}
\hline \multirow{2}{*}{ Statistical values } & \multicolumn{3}{|c|}{$\%$ Changes in actual (a) values from virtual (v) values } \\
\hline & $\left(\mathrm{a} E_{\max }-\mathrm{v} E \max \right) / \mathrm{v} E_{\max }$ & $(\mathrm{aPE}-\mathrm{vPE}) / \mathrm{vPE}$ & $(a P V A-v P V A) / v P V A$ \\
\hline \multicolumn{4}{|l|}{ Mean } \\
\hline Mean \pm SD (CV) & $-0.38 \pm 0.49(1.3)$ & $-0.72 \pm 0.43(0.6)$ & $-0.12 \pm 0.06(0.5)$ \\
\hline$p$ & $0.12(\mathrm{~ns})$ & $0.009(<0.05)$ & $0.003(<0.05)$ \\
\hline \multicolumn{4}{|l|}{ Median } \\
\hline Mean \pm SD (CV) & $0.31 \pm 1.44(4.6)$ & $0.12 \pm 1.67(14)$ & $0.05 \pm 0.33(6.6)$ \\
\hline$p$ & $0.63(\mathrm{~ns})$ & $0.87(\mathrm{~ns})$ & 0.75 (ns) \\
\hline \multicolumn{4}{|l|}{ Mode } \\
\hline Mean \pm SD $(C V)$ & $0.11 \pm 2.98(27)$ & $0.01 \pm 2.97(297)$ & $0.05 \pm 1.05(21)$ \\
\hline$p$ & $0.93(\mathrm{~ns})$ & 0.99 (ns) & 0.98 (ns) \\
\hline \multicolumn{4}{|l|}{ SD } \\
\hline Mean \pm SD (CV) & $-13.6 \pm 3.05(0.2)$ & $-4.8 \pm 12.35(2.6)$ & $2.3 \pm 1.15(0.5)$ \\
\hline$p$ & $0.0001(<0.05)$ & 0.38 (ns) & $0.005(<0.05)$ \\
\hline \multicolumn{4}{|l|}{ Variance } \\
\hline Mean \pm SD (CV) & $-25.2 \pm 5.3(0.2)$ & $-8.1 \pm 24.6(3.0)$ & $4.7 \pm 2.4(0.5)$ \\
\hline$p$ & $0.0001(<0.05)$ & $0.46(\mathrm{~ns})$ & $0.005(<0.05)$ \\
\hline \multicolumn{4}{|l|}{ Range } \\
\hline Mean \pm SD (CV) & $-13.3 \pm 3.35(0.3)$ & $-3.78 \pm 14.85(3.9)$ & $1.83 \pm 1.03(0.6)$ \\
\hline$p$ & $0.0002(<0.05)$ & $0.56(\mathrm{~ns})$ & $0.007(<0.05)$ \\
\hline \multicolumn{4}{|l|}{ Minimum } \\
\hline Mean \pm SD (CV) & $12.3 \pm 6.35(0.5)$ & $2.93 \pm 10.51(3.6)$ & $-4.76 \pm 4.87(1.0)$ \\
\hline$p$ & $0.005(<0.05)$ & $0.61(\mathrm{~ns})$ & 0.06 (ns) \\
\hline \multicolumn{4}{|l|}{ Maximum } \\
\hline Mean \pm SD (CV) & $-6.42 \pm 2.98(0.5)$ & $-4.58 \pm 5.86(1.3)$ & $0.48 \pm 0.20(0.4)$ \\
\hline$p$ & $0.003(<0.05)$ & 0.11 (ns) & $0.002(<0.05)$ \\
\hline
\end{tabular}

$\mathrm{CV}$ (=SD/Mean): coefficient of variation. $p$ for paired t test with respect to zero. $\mathrm{ns}: p>0.05$.

greater than $\mathrm{v} E_{\max }$ at a smaller $E_{\max }$ level than control. Simultaneously, however, aPVA is greater than vPVA by the triangular area labeled (i) at a greater $E_{\max }$ than control and smaller than vPVA by the triangular area (ii) at a smaller $E_{\text {max }}$ level than control (Fig. 3).

These actual (prefix: a) and virtual (prefix: v) parameters could theoretically or geometrically be related to the given $V_{\mathrm{d}}$ and $P_{\mathrm{d}}$ and the measured Ves and Pes as follows.

$$
\begin{gathered}
\mathrm{v} E_{\text {max }}=P_{\mathrm{es}} /\left(V_{\mathrm{es}}-\mathrm{v} V_{\mathrm{o}}\right) \\
V_{\mathrm{d}}=\mathrm{v} V_{\mathrm{o}}+P_{\mathrm{d}} / \mathrm{v} E_{\text {max }} \\
\mathrm{a} E_{\max }=\left(P_{\mathrm{es}}-P_{\mathrm{d}}\right) /\left(V_{\mathrm{es}}-V_{\mathrm{d}}\right) \\
\mathrm{a} V_{\mathrm{o}}=V_{\mathrm{d}}-P_{\mathrm{d}} / \mathrm{a} E_{\max } \\
\mathrm{a} P V A=\mathrm{a} P E+E W
\end{gathered}
$$

These equations are inset in Fig. 3.

Surgical preparation. The details of the canine heart preparation were described in our previous papers $[9-11,14,17]$. Briefly, we performed the canine experiments in conformity with the guiding principles for the care and use of animals in the field of physiological sciences of the Japan Physiological Society and the American Physiological Society. We anesthetized six mongrel dogs (6-12 kg) with pentobarbital sodium $(25 \mathrm{mg} / \mathrm{kg}$, I.V.) after premedication with ketamine hydrochloride $(50 \mathrm{mg} /$ $\mathrm{kg}$, I.M.) and intubated them in each experiment. We maintained the anesthesia by fentanyl $(100 \mu \mathrm{g} / \mathrm{h}$ per dog, I.V.) as usual.

After opening the chest of the dog midsternally, we inserted a $3 \mathrm{~F}$ catheter-tip micromanometer into the $\mathrm{LV}$ from the apex to measure the LV pressure $(P)$. We introduced a 7F eight-electrode conductance catheter (Webster Laboratories, Baldwin Park, CA, USA) into the LV through an apical stab and placed it along the ventricular long axis to measure LV volume $(V)$ continuously. Our previous papers described in detail the method for measuring LVV with this catheter [9$11,14,17]$.

Ventricular volumetry. Briefly, with this catheter we continuously measured the time-varying electrical conductance of the blood in the LV cavity. We used our custom-made signal conditioner-processor (SI 
$E_{\max }$ and PVA Distribute Normally under AF

Table 2. Normal frequency distributions of $E_{\max }, \mathrm{PE}$, and PVA despite $E_{\max }$ dependency of $V_{\mathrm{o}}$.

\begin{tabular}{cccc}
\hline \multirow{2}{*}{ Normality measures (dimensionless) } & \multicolumn{3}{c}{ Actual (a) $E_{\max }, \mathrm{PE}$, and PVA data } \\
\cline { 2 - 4 } & $\mathrm{a} E_{\max }$ & $\mathrm{aPE}$ & $\mathrm{aPVA}$ \\
\hline Kurtosis & $-0.64 \pm 0.41$ & $1.35 \pm 1.86$ & $-0.41 \pm 0.47$ \\
Mean $\pm \mathrm{SD}$ & $0.012(<0.05)$ & $0.14(\mathrm{~ns})$ & $0.09(\mathrm{~ns})$ \\
$p$ & & & $0.08 \pm 0.49$ \\
Skewness & $-0.16 \pm 0.55$ & $0.97 \pm 0.30$ & $0.71(\mathrm{~ns})$ \\
Mean \pm SD & $0.52(\mathrm{~ns})$ & $0.0006(<0.05)$ & $2.24 \pm 1.55$ \\
$\chi^{2}$ & & & $0.16-0.99(\mathrm{~ns})$ \\
Mean $\pm \mathrm{SD}$ & $3.00 \pm 1.89$ & $2.03 \pm 0.90$ & $0.36-0.999(\mathrm{~ns})$ \\
$P$ & $0.071-0.77(\mathrm{~ns})$ &
\end{tabular}

For kurtosis and skewness, $p$ for paired t test with respect to 0 . For $\chi^{2}$ test, the full range of $p$ is shown. ns: $p>0.05$.

Medicotech Co., Ltd., Japan) to convert the blood conductance to LV volume (LVV). For more details, refer to our previous paper [10].

Unstressed volume or volume-axis intercept $\left(\mathbf{V}_{\mathrm{o}}\right)$. We used $E_{\max }$ as a reliable, load-independent index of LV contractility that was originally proposed by Suga and has been generally accepted [18, 21, 23]. To calculate $E_{\max }$ of every beat, we needed the V-axis intercept $\left(V_{\mathrm{o}}\right)$ of the ESPVR line (Figs. 1-3).

We determined an ESPVR and $V_{\mathrm{o}}$ by clamping the inferior vena cava over $\sim 10$ regular beats before the first AF as usual [9-11, 14, 17]. This clamp gradually decreased both LVP and LVV and shifted the shrinking LV P-V loop toward the origin of the P-V diagram. We drew a straight line tangential to or through the end-systolic, left-upper corners of these P-V loops and extrapolated the line to the $\mathrm{V}$ axis to obtain its $V_{0}$. These ESPVR and $V_{\mathrm{o}}$ are their virtual values (vESPVR, $v V_{\mathrm{o}}$ ) to yield the virtual $E_{\text {max }}$ values $\left(\mathrm{v} E_{\text {max }}\right)$ in the conventional method [10] (Fig. 3).

Contractility index $\left(E_{\max }\right)$. After we obtained the conventional virtual $E_{\max }\left(\mathrm{v} E_{\max }\right)$ by Eq. 1 in Fig. 3, we newly obtained $\mathrm{a} E_{\max }$ by Eq. 3 in Fig. 3. We first calculated $V_{\mathrm{d}}$ from $\mathrm{v} V_{\mathrm{o}}, \mathrm{v} E_{\text {max }}$, and $P_{\mathrm{d}}$ by Eq. 2 in Fig. 3. Here, we assumed $P_{\mathrm{d}}=-10 \mathrm{~mm} \mathrm{Hg}$ as a reasonably representative value close to the mean $P_{\mathrm{d}}(9.5 \pm 2.0$ $\mathrm{mmHg}$ ) empirically obtained in our previous study [22]. Once $\mathrm{a} E_{\max }$ was determined, we obtained $\mathrm{a} V_{\text {o }}$ by Eq. 4 in Fig. 3. The a $V_{\mathrm{o}}$ is needed to calculate the aPVA.

Pressure-volume area (PVA). PVA consists of the two areas (Fig. 1): the external mechanical work (EW) (rectangular area) within the P-V loop and the elastic potential energy (PE) (triangular area) between the end-systolic $\mathrm{P}-\mathrm{V}$ or $E_{\max }$ line and the end-diastolic $\mathrm{P}-\mathrm{V}$ curve on the origin side of the P-V loop. These two areas of each sampled beat were calculated in a computer as explained previously in detail [10]. PE had a difference between vPE and aPE by the area marked as (1) or (2) in Fig. 3. In the $E_{\max }$ range higher than the control, $\mathrm{a} E_{\max }$ was smaller than $\mathrm{v} E_{\max }$ and area (1) had to be added to PE and then to PVA to obtain aPE and aPVA. In the $E_{\max }$ range lower than the control, a $E_{\text {max }}$ was larger than $\mathrm{v} E_{\max }$ and area (2) had to be subtracted from PE and then from PVA to obtain aPE and aPVA.

Atrial fibrillation. We attached a pair of stimulation electrodes to the left atrial appendage. Suprathreshold electrical stimulation at $20 \mathrm{~Hz}$ via these electrodes induced and maintained AF [9-11, 14, 17]. We produced AF for 2 min and recorded LV P and $\mathrm{V}$ during the latter $1 \mathrm{~min}$ at sampling intervals of $3 \mathrm{~ms}$ in a computer.

Statistics. We performed the basic statistical analyses of $\mathrm{a} E_{\max }, \mathrm{aPE}$, and aPVA of individual arrhythmic beats sampled in one min under AF in each heart (Table 1). We studied their frequency distributions not only by the two dimensionless measures of departure from normality or Gaussianity: kurtosis and skewness, but also by a $\chi^{2}$ test of their normality, evaluating this test by its $p$ value (Table 2) [10, 24, 25]. Zero kurtosis and skewness indicate the normality of the frequency distribution [10, 24]. A positive kurtosis indicates a sharper peak and a negative kurtosis a duller peak than the normality $[6,11]$. A positive skewness indicates a leftward shift of the peak frequency and a negative skewness its rightward shift $[10,24]$. A $\chi^{2}$ test supports the normality of the frequency distribution when its $\mathrm{p}$ value is greater than $0.05[10,24]$. We compared these statistical results of $\mathrm{a} E_{\max }, \mathrm{aPE}$, and aPVA with those of $\mathrm{v} E_{\max }, \mathrm{vPE}$, and vPVA (Table 1 ). In these statistical analyses, we used both Microsoft Office 2001 Excel and Stat View version 4.5. The data were presented as mean $\pm \mathrm{SD}$. 


\section{S. MOHRI et al.}
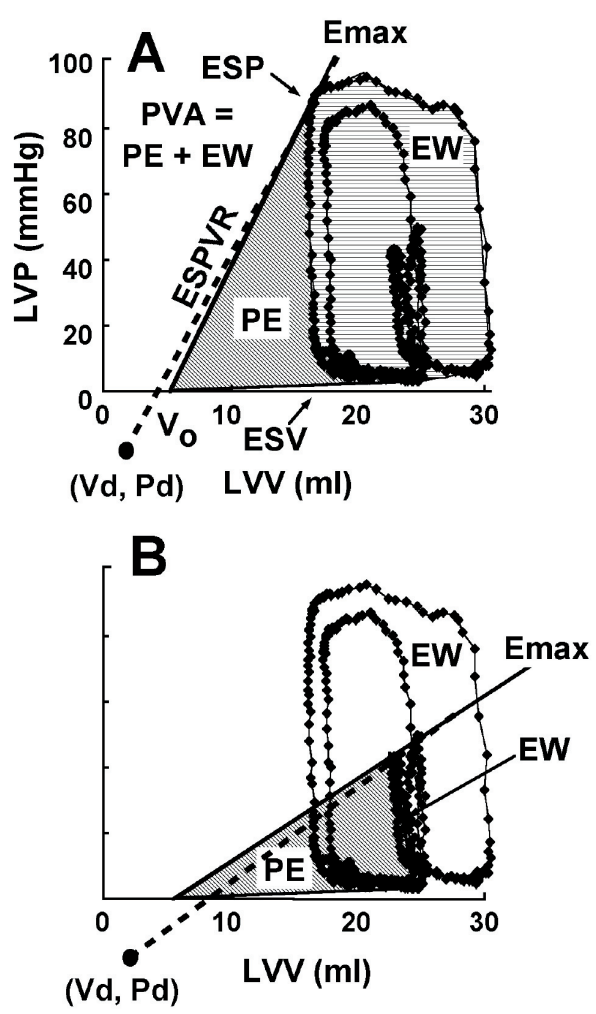

Fig. 4. Representative examples of the effects of the $E_{\max }$-dependent changes in $V_{0}$ on the ESPVR lines and the resultant shifts of the ESPVR lines. Panel A shows a contraction with a relatively high $E_{\max }$. Panel $B$ shows another with a relatively low $E_{\max }$. In Panel $A$, the hatched area for the elastic potential energy (PE) increases by the white area between the original solid-line ESPVR and the new dashed-line ESPVR. In Panel B, PE decreases by the hatched area between the original solid-line ESPVR and the new dashed-line ESPVR within the PE area. The area (horizontally shaded in Panel A or virtually zero in Panel B) for external mechanical work (EW) remains unchanged in either Panel A or B.

\section{RESULTS}

The artificial AF produced LV absolute arrhythmia at heart rates of $223 \pm 36(\mathrm{SD})$ beats/min in the six LVs, although these were significantly $(p<0.05)$ higher than their regular pacing rates of $169 \pm 15$ beats/min as previously reported [9]. Under AF, the RR1/RR2 of arrhythmic beats varied from $0.82 \pm 0.07$ at minimum to $1.91 \pm 0.27$ at maximum, and the maximum/minimum ratio was $2.35 \pm 0.45$. Here, RR1 is the beat interval preceding the beat whose $\mathrm{E}_{\max }$ is of interest, and RR2 the beat interval preceding the RR1, which determines proportionally the contractile potentiation., whereas RR2 determines inversely the contractile restitution [17]. Simultaneously, the maximum/minimum ratio of $E_{\max }$ was $3.75 \pm 0.90$, though this $E_{\max }$ corresponds to $\mathrm{v} E_{\max }^{\max }$ in the present study.
Figure 4 shows the same representative set of LV $\mathrm{P}-\mathrm{V}$ loops of 4 consecutive arrhythmic beats under $\mathrm{AF}$ in both panels. Panel A illustrates $E_{\max }, \mathrm{PE}$, and EW of the arrhythmic beat with the largest $E_{\max }$ and PVA of these four beats. Panel B illustrates those of the arrhythmic beat with the smallest $E_{\max }$ and PVA of these four beats. Of the pair of ESPVRs in each Panel, the solid line shows VESPVR and the dashed one aESPVR. The two vESPVRs in both panels had the same $\mathrm{v} V_{0}$. In contrast, the two aESPVRs connecting the $V_{\mathrm{d}}-P_{\mathrm{d}}$ point (solid circle) below the $\mathrm{V}$ axis had different $\mathrm{a} V_{\mathrm{o}}$ 's. In Panel A, a $V_{\mathrm{o}}$ was smaller than $\mathrm{v} V_{\mathrm{o}}$, but in Panel $\mathrm{B}, \mathrm{a} V_{\mathrm{o}}$ was greater than $\mathrm{v} V_{\mathrm{o}}$, though not labeled as a $V_{0}$ and $\mathrm{v} V_{0}$.

In Panel A, PVA consists of the sum of the quasirectangular (horizontally shaded) area within the P-V loop and the quasi-triangular (hatched) area between the vESPVR line and the EDPVR curve on the origin side of the $\mathrm{P}-\mathrm{V}$ loop of this contraction. The rectangular area represents the EW that the LV performed by ejecting blood into the aorta in this contraction. The triangular area represents the PE that this contraction generated in the LV wall, but was not converted into EW $[21,23]$. The EW was not influenced by the difference between the vESPVR and aESPVR. However, the PE was greater under the aESPVR than under the vESPVR by the small triangular area, corresponding to area (1) between the vESPVR and aESPVR in Fig. 3.

In Panel B, the EW was almost zero because of virtually zero stroke volume, and not influenced by the difference between vESPVR and aESPVR. However, the PE was smaller under the aESPVR than under the vESPVR by the small triangular area, corresponding to area (2) between the vESPVR and aESPVR in Fig. 3 . Thus, not only $\mathrm{a} E_{\text {max }}$ but also aPVA, predictably changed with the $E_{\max }$-dependent a $V_{0}$ changes.

Figure 5 summarizes the result of our analysis of $\mathrm{a} V_{0}$. The $\mathrm{v} V_{\mathrm{o}}$ of the stable regular contractions (open circle) was $26.1 \pm 4.9 \mathrm{ml} / 100 \mathrm{~g} \mathrm{LV}$ in the present six hearts. Under the reasonable assumption of $\mathrm{Pd}$ of $-10 \mathrm{mmHg}$ as described above [22], $V_{\mathrm{d}}$ obtained by extrapolating the vESPVR line to this $P_{\mathrm{d}}$ in the stable regular contractions was $25.3 \pm 5.1 \mathrm{ml} / 100 \mathrm{~g} \mathrm{LV}$ in these hearts. These $V_{\mathrm{d}}$ values were smaller than the $\mathrm{v} V_{\mathrm{o}}$ values of the stable regular contractions in the respective LVs by $0.83 \pm 0.40 \mathrm{ml} / 100 \mathrm{~g} \mathrm{LV}(>0, p<$ $0.0005)$, ranging from 0.40 to $1.40 \mathrm{ml} / 100 \mathrm{~g} \mathrm{LV}$. Each aESPVR line connects the thus determined $V_{\mathrm{d}}-P_{\mathrm{d}}$ point (large solid circle) and the end-systolic P-V point of the P-V loop of a given arrhythmic contraction. The intercept of each aESPVR line on the $\mathrm{V}$ axis is the $\mathrm{a} V_{\text {o }}$ 


\section{$E_{\max }$ and PVA Distribute Normally under AF}



Fig. 5. Experimental data of mean \pm SD and range (in parentheses) of $a E_{\max }$ and $a V_{\circ}$ (smaller solid circles) shown in a relatively low LVP range of the $\mathbf{P}-\mathbf{V}$ diagram. $a V_{0}$ and $\checkmark V$ (open circle) values are shown in terms of $a V-V$ and $\checkmark V_{0}-V_{d}$ as indicated by braces, since both $E_{\max }$ and PVA of our present interest are determined by such corrected $a V$ and $v V$. The three dashed lines are aESPVR lines. The larger solid circle is the $V_{d}-P_{d}$ point as the fulcrum of aESPVR lines.

of the respective contraction. The a $V_{0}$ varied with the arrhythmic changes in $E_{\max }$. The a $V_{\mathrm{o}}$ thus obtained as the consequence of its $E_{\text {max }}$ dependency was $26.2 \pm 4.9$ $\mathrm{ml} / 100 \mathrm{~g} \mathrm{LV}$ on average (range: 20.0-30.4 ml/100 g $\mathrm{LV}$ ) in the six hearts. The mean a $V$ was insignificantly greater than the $\mathrm{v} V$ by $0.06 \pm 0.09 \mathrm{ml} / 100 \mathrm{~g} \mathrm{LV}$ ( $p=$ $0.18)$. Figure 5 further shows the magnitudes of $\mathrm{a} V$ values above $V_{\mathrm{d}}$ at the maximum and minimum a $E_{\max }$ levels in the working range under AF.

We then compared the statistical values, such as mean, SD, variance, range, minimum, and maximum values of $\mathrm{a} E_{\text {max }}, \mathrm{aPE}$, and aPVA obtained in the present study with those of their virtual values ( $E_{\max }, \mathrm{vPE}$, and vPVA). Table 1 summarizes the percent changes (mean $\pm \mathrm{SD}$ and coefficient of variation: $\mathrm{CV}=\mathrm{SD} /$ mean) of the individual statistical values of $E_{\text {max }}$, $\mathrm{PE}$, and $\mathrm{PVA}\left(\Delta E_{\max }, \Delta \mathrm{PE}\right.$, and $\Delta \mathrm{PVA}$, where $\Delta=$ actual - virtual) by the $E_{\max }$-dependent a $V_{\mathrm{o}}$ changes. Although the mean, median, and mode of aEmax were not significantly different from those of $\mathrm{v} E_{\max }$, its $\mathrm{SD}$, variance, range, and maximum values of $\mathrm{a} E_{\max }$ were significantly smaller than those of $\mathrm{v} E_{\max }$, whereas only the minimum value of $\mathrm{a} E_{\max }$ was significantly greater than that of $\mathrm{v} E_{\max }$. As the consequence of these $\mathrm{a} E_{\max }$ and $\mathrm{a} V$ changes in individual arrhythmic beats, aPE and aPVA also changed significantly, but only slightly from the corresponding vPE and vPVA. For aPE, only the slight decrease in its mean was significant, but the changes in its median, mode, SD, variance, range, minimum, and maximum were little and insignificant. As for aPVA, the changes in its mean, SD, variance, range, and maximum were significant, but the changes in its median, mode, and minimum were only slight, and they were insignificant. These significant changes were opposite in direction between $\Delta E_{\text {max }}$ and $\Delta \mathrm{PVA}$.

The SD, variance, range, and maximum of the percent $\triangle \mathrm{PVA}$ were significant, although those of percent $\triangle \mathrm{PE}$ were insignificant (Table 1). The significant $\triangle \mathrm{PVA}$ changes accompanied the $\mathrm{CV}$ (coefficient of variation, $\mathrm{SD} /$ mean) values smaller than 1 , whereas the insignificant $\triangle \mathrm{PE}$ changes accompanied the $\mathrm{CV}$ values greater than 1 .

Figure 6 compares a representative pair of the frequency distributions of the $\mathrm{v} E_{\max }$ and $\mathrm{vPVA}$ using $\mathrm{v} V_{\mathrm{o}}$ (Panels $\mathrm{A}$ and $\mathrm{C}$ ) and the aEmax and aPVA using $\mathrm{a} V_{\mathrm{o}}$ (Panels B and D) in one LV. The insets list their kurtosis, skewness, and $\chi^{2}\left(p>0.685\right.$ for $\mathrm{a} E_{\max }$ and $\mathrm{v} E_{\max }, p>0.999$ for aPVA and vPVA). These values support the normality or Gaussianity of these four distributions. There were little differences of these statistical results between the respective pairs of the virtual and actual distributions. Although not shown, neither vPE nor aPE were very different. The other 5 LVs had essentially similar results.

Table 2 summarizes the mean \pm SD of kurtosis, skewness, and $\chi^{2}$ values of aEmax, aPE, and aPVA with their $p$ values in the six hearts. All the kurtosis, skewness, and $\chi^{2}$ values supported the normality of the frequency distribution of aPVA.

\section{DISCUSSION}

Our present statistical study evidently shows that the normal or Gaussian frequency distribution of not only $E_{\max }$ but also PVA, remained unchanged even if we took the $E_{\max }$-dependent $V_{\text {o }}$ changes into consideration.

We previously reported the normality of the frequency distributions of both $\mathrm{v} E_{\text {max }}$ and $\mathrm{vPVA}$ of the absolute arrhythmic beats under AF in the canine hearts despite the significantly non-normal or non-Gaussian frequency distributions of the cardiodynamic variables serving as the determinants of PVA [10]. Of these, the most non-normally distributing variable proved to be Ea and the next to be the RR beat interval [10]. However, both $E_{\text {max }}$ and PVA could remain normal very likely because of the central limit theorem, as we speculated previously [10]. In the present study, Ea remained unchanged despite the $E_{\max }$ dependency of $V_{\text {o }}$ (Fig. 1). Moreover, $E_{\max }$ was only slightly, though significantly, affected by the $E_{\max }$-dependent $V_{\mathrm{o}}$, much less than we considered (Table 1, Fig. 6). As the result, 

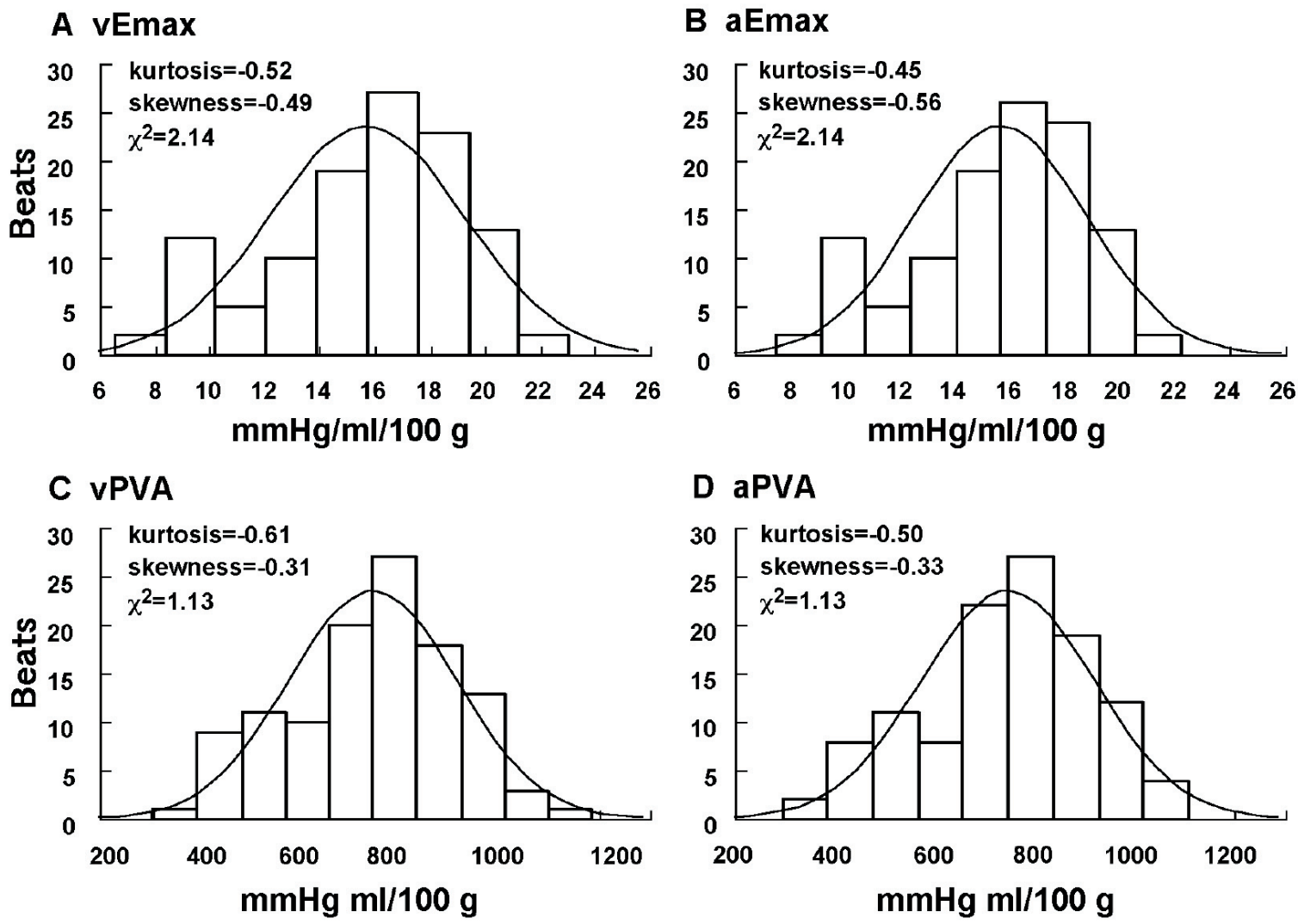

D APVA

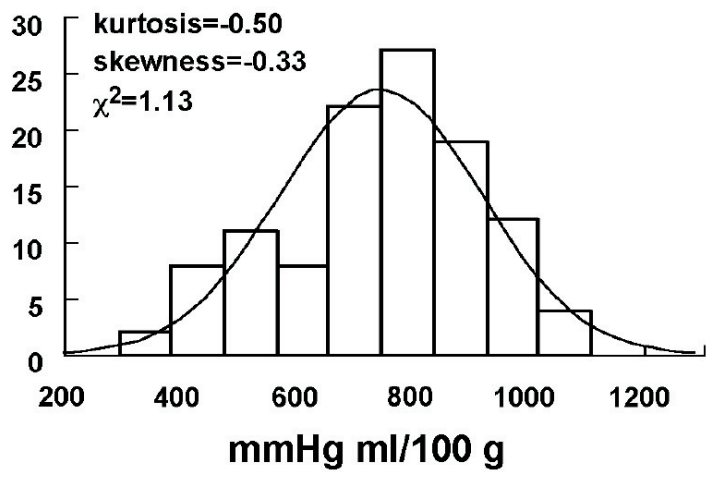

Fig. 6. Comparison of the frequency distribution histograms of vPVA (virtual PVA), using a constant $v V_{\text {o (virtual }} V_{0}$ ) in Panel $\mathrm{A}$, and aPVA (actual PVA), using an $E_{\max }$-dependent a $V_{\text {o }}$ (actual $V_{\circ}$ ) in Panel $B$ in one heart as a representative case. A continuous set of 113 beats/30 s were analyzed. The curves are the bestfit normal or Gaussian distribution curves. Although all of

the normality of the frequency distribution of $E_{\text {max }}$ in terms of $\mathrm{a} E_{\max }$ were little affected.

The slight dependency of a $E_{\max }$ on the $E_{\max }$-dependent $\mathrm{a} V_{\mathrm{o}}$ seems to have led to the slight dependency of aPVA on the a $V_{0}$. This is accountable by the little effect of the $E_{\max }$-dependent $\mathrm{a} V_{\mathrm{o}}$ on the aPE part of the aPVA and no effect on the EW part of the aPVA (Figs. 1 and 3).

One may wonder why the SD, variance, range, and maximum of the percent $\triangle \mathrm{PVA}$ were significant, although those of percent $\triangle \mathrm{PE}$ were insignificant (Table 1). This seems to have derived from the following two relations: $\mathrm{PVA}=\mathrm{EW}+\mathrm{PE}$ and $\triangle \mathrm{PVA}=$ $\triangle \mathrm{PE}$ where $\mathrm{EW}$ is always independent of $V_{\mathrm{o}}$ changes (Fig. 4). Since PE is always smaller than PVA by a constant $\mathrm{EW}$, the percent $\triangle \mathrm{PVA}(\triangle \mathrm{PVA} / \mathrm{PVA}=\Delta \mathrm{PE} /$ $\mathrm{PVA})$ is always smaller than the percent $\triangle \mathrm{PE}(\triangle \mathrm{PE} / \mathrm{PE}$ $=\Delta \mathrm{PE} /[(\mathrm{PVA}-\mathrm{EW})])$ in each arrhythmic beat. This seems to have caused the present statistical differences of the SD, variance, range, and maximum between the percent $\triangle \mathrm{PVA}$ and $\triangle \mathrm{PE}$.

the mean, median, mode, SD, variance, range, maximum, and minimum values of either $a E_{\text {max }}$ or aPVA slightly but significantly changed from those of $V P V A$ as shown in Table 1 , their kurtosis, skewness, and $\chi^{2}$ values $(p>0.05)$ as inset here were significantly different neither between $\vee E_{\max }$ and $\mathrm{a} E_{\max }$ nor between VPVA and aPVA.

Figure 7 shows that we could attribute the relatively small changes in not only a $E_{\max }$ but also aPVA, to the hyperbolic a $V_{\mathrm{o}}-\mathrm{a} E_{\max }$ relation. This hyperbolic relation shows the $E_{\max }$ dependency of $V_{\mathrm{o}}$ as represented by the inset formula: $\mathrm{a} V_{\mathrm{o}}=V_{\mathrm{d}}-P_{\mathrm{d}} / \mathrm{a} E_{\max }$ (Eq. 4 in Fig. 3). $\mathrm{a} V_{\mathrm{o}}$ increases slightly even with considerable decreases in $\mathrm{a} E_{\max }$ from 30 to $10 \mathrm{mmHg} /(\mathrm{ml} / 100 \mathrm{~g} \mathrm{LV})$. However, $\mathrm{a} V_{\mathrm{o}}$ increases moderately with further decreases in $\mathrm{a} E_{\text {max }}^{\circ}$ to below $10 \mathrm{mmHg} /(\mathrm{ml} / 100 \mathrm{~g} \mathrm{LV})$, and then markedly with further decreases in $\mathrm{a} E_{\max }$ below 5 $\mathrm{mmHg} /(\mathrm{ml} / 100 \mathrm{~g} \mathrm{LV})$. As described in Results, the maximum/minimum ratio of $\mathrm{v} E_{\max }$ was $\sim 4$ on average in the six hearts. The maximum/minimum ratio of $\mathrm{a} E_{\max }$ was $\sim 13 \%$ smaller on average than that of $\mathrm{v} E_{\max }$, as shown in Table 1 and by the horizontal bi-head arrows and the thick solid line on the abscissa in Fig. 7.

Figure 7 also shows that the same tendency holds whether $P_{\mathrm{d}}$ was set $5 \mathrm{mmHg}$ either higher or lower than $-10 \mathrm{mmHg}$ (dashed curves). As a result, the 
$E_{\max }$ and PVA Distribute Normally under AF

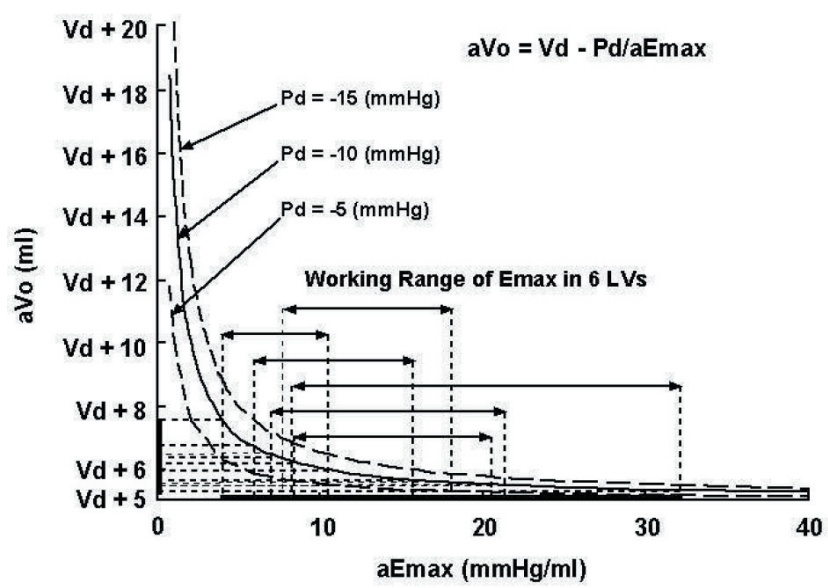

Fig. 7. The theoretically inverse relations (inset equation) between $a E_{\max }$ (actual $E_{\max }$ ) and a $V_{0}$ (actual $V_{0}$ ) that account for the relatively small changes in a $V_{\text {o }}$ (thick part of the ordinate) despite the large changes in $a E_{\max }$ (thick part of the abscissa) among the arrhythmic contractions under atrial fibrillation. The solid curve is for $P_{d}=-10 \mathrm{mmHg}$. The dashed curves on both sides of the solid curve are for $P_{d}= \pm 5 \mathrm{mmHg}$ around $-10 \mathrm{mmHg}$. The horizontal bi-head arrows show the working ranges of $a E$ ${ }_{\text {max }}$ in the six individual LVs, where the dashed vertical and horizontal lines show the maximum and minimum values of $\mathrm{a} E_{\max }$ and $a V_{0}$.

arrhythmic changes in $\mathrm{a} E_{\max }$ within the thick part of the abscissa change a $V_{0}$ only by a few $\mathrm{ml} / 100 \mathrm{~g} \mathrm{LV}$, but a further decrease in $\mathrm{a} E_{\text {max }}$ below the thick part of the abscissa can sensitively increase a $V_{0}$. However, the arrhythmic changes in $\mathrm{a} E_{\max }$ during $\mathrm{AF}$ did not fall below $\sim 5 \mathrm{mmHg} /(\mathrm{ml} / 100 \mathrm{~g} \mathrm{LV})$, enough to increase $\mathrm{a} V_{\mathrm{o}}$ and thus distort the normal distributions of $\mathrm{a} E_{\max }$ and aPVA.

We therefore conclude that even the variable a $V$ keeps the normal frequency distributions of both $\mathrm{a} E_{\max }$ and aPVA of arrhythmic LV contractions under AF because the $E_{\max }$-dependent $V_{\mathrm{o}}$ changes are too small to affect their distributions. Therefore, we can practically ignore the changes, if any, in $E_{\max }$ and PVA with the $E_{\max }$-dependent $V_{\text {o }}$ changes under $\mathrm{AF}$ in the statistical characterizations of arrhythmic contractions. Moreover, this evidently corroborates our previous conclusion [10].

This work was partly supported by Scientific Research Grants (15650095, 15659186, 15700330, 16659057) from the Ministry of Education, Culture, Sports, Science and Technology, and Cardiovascular Diseases Research Grants (14A-1) from the Ministry of Health, Labour and Welfare, both of Japan.

\section{REFERENCES}

1. Atwood JE, Myers J, Sandhu S, Lachterman B, Friis R, Oshita A, Forbes S, Walsh D, and Froelicher V: Optimal sampling interval to estimate heart rate at rest and under exercise in atrial fibrillation. Am J Cardiol 63: 45-48, 1989

2. Bootsma BK, Hoelen AJ, Strackerr J, and Meijler FL: Analysis of RR intervals in patients with atrial fibrillation at rest and during exercise. Circulation 41: 783-793, 1970

3. Brookes CI, White PA, Staples M, Oldershaw PJ, Redington AN, Collins PD, and Noble MI: Myocardial contractility is not constant during spontaneous atrial fibrillation in patients. Circulation 98: 1762-1768, 1998

4. Daoud EG, Weiss R, Bahu M, Knight BP, Bogun F, Goval R, Harvey M, Strickberger SA, Man KC, and Morady F: Effect of an irregular ventricular rhythm on cardiac output. Am J Cardiol 78: 1433-1436, 1996

5. Dubrey SW and Falk RH: Optimal number of beats for the Doppler measurement of cardiac output in atrial fibrillation. J Am Soc Echocardiogr 10: 67-71, 1997

6. Hardman SMC, Noble MIM, and Seed WA: Postextrasystolic potentiation and its contribution to the beat-tobeat variation of the pulse during atrial fibrillation. Circulation 86: 1223-1232, 1992

7. Hoopen MT and Bongaarts JPM: Probabilistic characterization of R-R intervals. Cardiovasc Res 3: 218-226, 1969

8. Horan LG and Kistler JC: Study of ventricular response in atrial fibrillation. Circ Res 9: 305-312, 1961

9. Mitani H, Yamaguchi H, Morita T, Oshima Y, Shimizu J, Ito H, Araki J, Takaki M, Sano S, and Suga H: Effective arterial elastance of irregular beats during atrial fibrillation in canine left ventricle. Jpn J Physiol 50: 77-89, 2000

10. Mohri S, Shimizu J, Iribe G, Ito H, Morita T, Yamaguchi $H$, Sano S, Kajiya F, and Suga H: Normal distribution of ventricular pressure-volume area of arrhythmic beats during atrial fibrillation in canine heart. Am J Physiol Heart Circ Physiol. 288: H1740-H1746, 2005

11. Morita T, Araki J, Oshima $Y$, Mitani H, Iribe G, Mohri S, Shimizu J, Sano S, Kajiya F, and Suga H: Frequency distribution, variance, and moving average of left ventricular rhythm and contractility during atrial fibrillation in dog. Jpn J Physiol 52: 41-49, 2002

12. Muntinga HJ, Gosselink AT, Bianksma PK, De Kam PJ, Van Der Wall EE, and Crijns HJ: Left ventricular beat to beat performance in atrial fibrillation: dependence on contractility, preload, and afterload. Heart 82: 575-580, 1999

13. Rawles JM and Rowland E: Is the pulse in atrial fibrillation irregularly irregular? Br Heart J 56: 4-11, 1986

14. Shimizu J, Mohri S, Iribe G, Ito H, Morita T, Yamaguchi $\mathrm{H}$, Sano $\mathrm{S}$, and Suga $\mathrm{H}$ : Predictability of $\mathrm{O}_{2}$ consumption from contractility and mechanical energy of absolute arrhythmic beats in canine heart. Jpn J Physiol 55: 135-142, 2005

15. Suzuki S, Araki J, Morita T, Mohri S, Mikane T, Yamaguchi $\mathrm{H}$, Sano S, Ohe T, Hirakawa M, and Suga H: Ventricular contractility in atrial fibrillation is predictable by me- 


\section{S. MOHRI et al.}

chanical restitution and potentiation. Am. J. Physiol. 275: H1513-H1519, 1998

16. Watt JH, Donner AP, McKinney CM, and Klein GJ: Atrial fibrillation: minimal sampling interval to estimate average rate. J Electrocardiol 17: 153-156, 1984

17. Yamaguchi $H$, Takaki M, Ito H, Tachibana $H$, Lee $S$, and Suga $\mathrm{H}$ : Pressure-interval relationship characterizes left ventricular irregular beat contractilities and their mean level during atrial fibrillation. Jpn J Physiol 47: 101-110, 1997

18. Sagawa K, Maughan L, Suga H, and Sunagawa K: Cardiac Contraction and the Pressure-Volume Relationship, Oxford Univ Press, New York, pp 232-298, 1988

19. Sunagawa K, Maughan WL, Burkhoff D, and Sagawa $\mathrm{K}$ : Left ventricular interaction with arterial load studied in isolated canine heart. Am J Physiol 245: H773-H780, 1983

20. Nozawa T, Yasumura Y, Futaki S, Tanaka N, Uenishi M, and Suga H: Efficiency of energy transfer from pressure-volume area to external mechanical work increases with contractile state and decreases with afterload in the left ventricle of the anesthetized closed-chest dog. Circulation 77: 1116-1124, 1988

21. Suga H: Ventricular energetics. Physiol Rev 70: 247277, 1990

22. Suga $H$, Yasumura $Y$, Nozawa T, Futaki $S$, and Tanaka $\mathrm{N}$ : Pressure-volume relation around zero transmural pressure in excised cross-circulated dog left ventricle. Circ Res 63: 361-372, 1988

23. Suga $H$ : Cardiac mechanics and energetics-from Emax to PVA. Front Med Biol Eng 2: 3-22, 1990

24. Glantz SA: Primer of Biostatistics, McGraw-Hill Book Co., New York, pp 217-221 \& 284-292, 1981

25. Snedecor GW and Cochran WG: Statistical Methods, 6th ed, lowa State Univ Press, Ames, lowa, 1971 